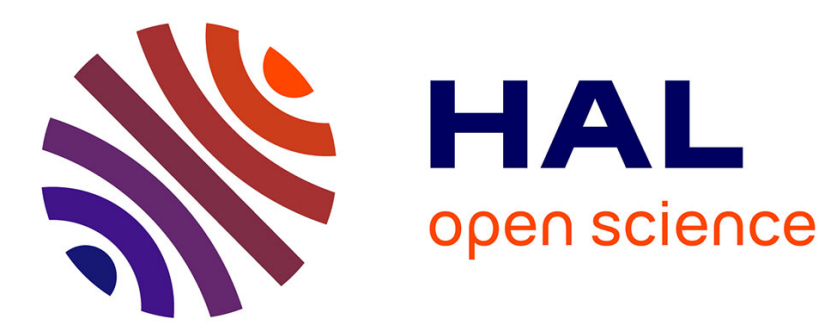

\title{
Hybrid imaging systems for depth of focus extension with or without post-processing
}

Frederic Diaz, François Goudail, Brigitte Loiseaux, Jean-Pierre Huignard

\section{To cite this version:}

Frederic Diaz, François Goudail, Brigitte Loiseaux, Jean-Pierre Huignard. Hybrid imaging systems for depth of focus extension with or without post-processing. 8th Euro-American Workshop on Information Optics, Jul 2009, Paris, France. pp.012009, 10.1088/1742-6596/206/1/012009 . hal-00749263

\section{HAL Id: hal-00749263 \\ https://hal-iogs.archives-ouvertes.fr/hal-00749263}

Submitted on 5 Apr 2013

HAL is a multi-disciplinary open access archive for the deposit and dissemination of scientific research documents, whether they are published or not. The documents may come from teaching and research institutions in France or abroad, or from public or private research centers.
L'archive ouverte pluridisciplinaire HAL, est destinée au dépôt et à la diffusion de documents scientifiques de niveau recherche, publiés ou non, émanant des établissements d'enseignement et de recherche français ou étrangers, des laboratoires publics ou privés. 


\title{
Hybrid imaging systems for depth of focus extension with or without post-processing
}

\author{
F. Diaz ${ }^{1,2}$, F. Goudail ${ }^{2}$, B. Loiseaux ${ }^{1}$, J.-P. Huignard ${ }^{1}$ \\ 1: Campus Polytechnique, 1 Avenue Augustin Fresnel, 91767 Palaiseau Cedex, France \\ 2 : Laboratoire Charles Fabry de l'Institut d'Optique, CNRS, Universite Paris-Sud, Campus \\ Polytechnique, RD 128, 91127 Palaiseau Cedex, France \\ E-mail: frederic.diaz@thalesgroup.com
}

\begin{abstract}
We address two different methods to enhance the depth of focus (DOF) according to the application, using amplitude and/or phase masks. The first application consists in increasing the length of the focal line of a lens while preserving its transverse resolution. We propose a mask inspired from holographic principles. We then address DOF enhancement with an hybrid imaging system composed of a pupil mask followed by a digital deconvolution step. We use an optimization criterion based on the final image quality to determine the optimal parameters of phase masks.
\end{abstract}

\section{Introduction}

Many types of pupil masks have been proposed for depth of focus (DOF) enhancement, with different properties and advantages which make them appropriate for different applications. The key point for choosing the optimal mask is to define the appropriate quality criterion related to the targeted application. The appropriate type of mask and the values of its parameters are then chosen to optimize this criterion. We will address two different types of applications. The first one consists in increasing the length of the focal line of a lens by using a novel type of mask inspired from holographic principles [1]. The second type of application concerns DOF enhancement in a hybrid imaging system composed of a pupil mask and a digital deconvolution step.

\section{Uniform extended focal line: the holographically generated complex mask}

The simplest designs to enhance the DOF are probably amplitude masks. Continuous phase masks and binary phase masks (BPM) can also be used, and they are usually considered more energy-efficient than amplitude masks since they have unit intensity transmission. However, in DOF extension, the relevant parameter is not the total transmitted energy, but the "useful" one, that is, the energy concentrated in the focal line. When this criterion is considered, amplitude/phase (complex) masks may have better performance.

We consider in this section that one wants to produce a light distribution as uniform as possible along the focal line. We define the depth of focus as the length of the region along the optical axis where the amplitude distribution has fluctuations lower than a given threshold. For given DOF and allowed fluctuations, our purpose is to determine the mask that maximizes the mean intensity in the DOF region. As an original alternative to the BPM [1], we propose 
a complex mask with the classical approach used for computer-generated holograms (Fig. 1). Such a design first specifies the desired normalized amplitude at chosen points along the optical axis, and then computes the mask that generates this distribution. This makes it possible to "tailor" the shape of the focal line. We will call this design "holographically generated complex mask" (HGCM) [2]. We achieved to obtain a DOF of $1.6 \mathrm{~mm}$ with intensity fluctuation below $1 \%$ with a lens with a focal length $f=160 \mathrm{~mm}$, an aperture $D=16 \mathrm{~mm}$, at a wavelength $\lambda=1064 n m$ (Fig. 2).

The lower transmission of the HGCM (Fig. 1) is compensated by a better concentration of energy in the DOF region, thanks to the contribution of amplitude part of the filter. Moreover, with the HGCM, it is possible to achieve an accurate localization of the DOF region. In our example, the DOF region is centred on the focal point, whereas the DOF region of the BPM is asymmetrically distributed. Further advantages of the HGCM are the sharper decrease of the intensity at the limit of the DOF region, lower sidelobes and the better invariance of the transverse spot size with respect to $z$ inside the DOF region.

The HGCM is thus a valuable alternative to the BPM for applications where very low fluctuations of the amplitude distribution along the optical axis are required and when the shape of the focal line has to be precisely tailored.
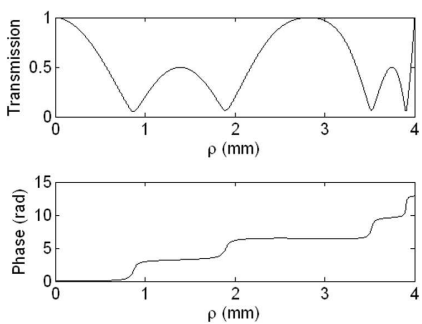

Figure 1. Profile of the HGCM
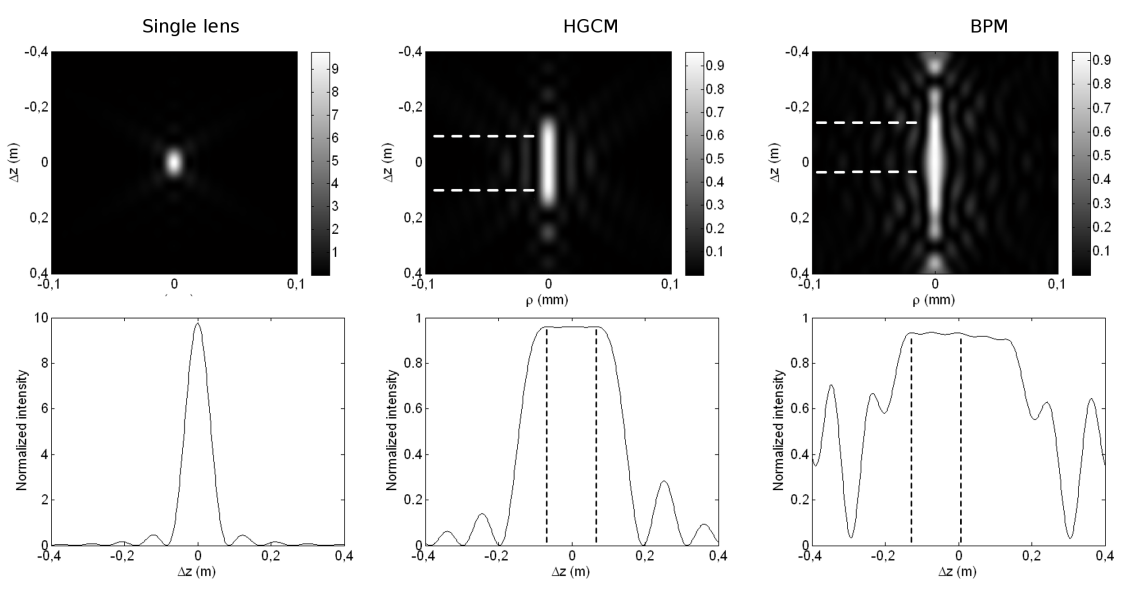

Figure 2. PSF of the lens, the BPM and the HGCM for a DOF of $1.6 \mathrm{~mm}$ and fluctuations below $1 \%$. The DOF region with variations below $1 \%$ are delimited with the dotted lines.

\section{DOF extension with hybrid imaging system: simultaneous optimization of the deconvolution and the pupil mask}

The approach discussed previously allows for moderate extension of the DOF while maintaining the transverse width of the point spread function (PSF) so that the resolution of the obtained image is little perturbed. When much higher DOF extension is required, the adequate masks tend to dramatically reduce the resolution. In this case, a deconvolution step is needed. In such hybrid imaging systems, two effects can degrade the quality of the final image: the variation of the PSF with respect to defocus, whereas the deconvolution filter is unique, and the noise enhancement due to deconvolution, which depends on the frequency response of the mask. Indeed, with usual phase masks, the more invariant to defocus the PSF is, the more they behave as low-pass filters. In such imaging systems, the relevant quality criterion is the quality of the final image obtained after the deconvolution step. We derived a mathematical expression of the output signal to noise ratio (SNR) that takes into account the detection noise and the defocus range where the system should provide an optimal image quality [3]. 
As an example, we consider the cubic phase mask [4] which is a pure phase mask whose PSF at the focal plane is $h(x, y)=|\mathrm{FT}\{P(x, y) \exp [i \phi(x, y)]\}|^{2}$ with $\phi(x, y)=\alpha \times\left(x^{3}+y^{3}\right), x$ and $y$ the coordinates of the pupil, normalized to $1, P(x, y)$ the pupil function, and $\alpha$ the phase excursion to be optimized. FT refers to the Fourier transform. The input SNR, defined by the SNR between the object and the noise of the sensor, is set at $S N R_{i n}=28 d B$ and the maximal defocus value $\psi_{\text {defocmax }}$ is 9.2 , with $\psi=\frac{\pi R^{2}}{\lambda}\left(\frac{1}{f}-\frac{1}{d_{O}}-\frac{1}{d_{I}}\right)$ where $R$ is the aperture radius, $f, d_{O}$ and $d_{I}$ are respectively the focal length, the object distance and the image sensor plane distance. The object is the classical "Lena" image.

The value of $S N R_{\text {out }}$ as a function of $\alpha$ is displayed in Fig 3. The optimal phase mask parameter is $\alpha_{\text {opt }}=10$, leading to $S N R_{\text {out }}=16.4 d B$. With lower $\alpha$, the PSF of the optical system is not insensitive enough to the defocus. With larger $\alpha$, the PSF is much insensitive to defocus, but the optical system has then a lower-pass behaviour and the noise is enhanced by the deconvolution step. The parameter $\alpha_{\text {opt }}=10$ is thus a compromise between theses two effects.

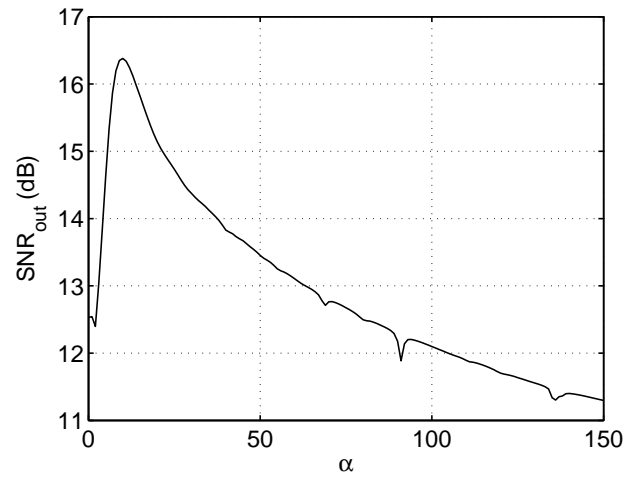

Figure 3. $S N R_{\text {out }}$ in function of $\alpha$ for Lena.

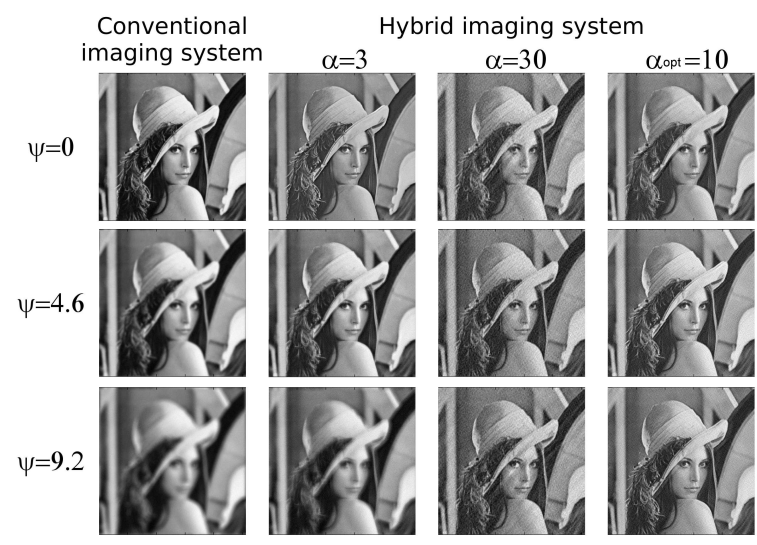

Figure 4. Results obtained with Lena with a conventional imaging system and with a hybrid imaging system at several values of $\alpha$.

As shown on the deconvoluted images in Fig 4, the choosen criterion, $S N R_{\text {out }}$, has an effective correlation with the visual perception.

\section{Conclusion}

As the relevant optimization criterion strongly depends on the targeted application, we have discussed two approaches to DOF extension that are adapted to different cases. For applications where an intensity extended focal line is desired, an appropriate optimization criterion is the energy along this focal line. For imaging systems, the optimisation criterion should be the final image quality for which the SNR is an appropriate criterion.

\section{References}

[1] L. Liu, F. Diaz, L. Wang, B. Loiseaux, J.-P. Huignard, C. J. R. Sheppard, N. Chen, "Superresolution along extended depth of focus with binary-phase filters for the Gaussian beam", J. Opt. Soc. Am. A 25, 2095 (2008)

[2] F. Diaz, F. Goudail, B. Loiseaux, J.-P. Huignard, "Design of a complex filter for depth of focus extension", Opt. Lett., 34, 1171 (2009).

[3] F. Diaz, F. Goudail, B. Loiseaux, J.-P. Huignard, "Increase in depth of field taking into account deconvolution by optimization of pupil mask, 34, (2009).

[4] W. Cathey, E. Dowski, "New paradigm for imaging systems", Appl. Opt, 41, 6080 (2002). 